\section{Rose bengal staining to evaluate ocular surface pathology in canine keratoconjunctivitis sicca}

\section{Alice Griffiths' ${ }^{2}$, David Williams ${ }^{1}$}

1 University of Cambridge, Cambridge, UK

2 Independent Vetcare, Bath, UK

The Schirmer tear test is the standard test to diagnose canine keratoconjunctivitis sicca (KCS). The STT measures tear production but says nothing directly about ocular surface pathology. In human ophthalmology Rose Bengal staining (RBS) is widely used to determine the health of the ocular surface in $\mathrm{KCS}$, yet in veterinary ophthalmology no research has evaluated RBS in canine KCS. We stained canine with and without KCS to determine the correlation of RBS with STT.

44 eyes of 24 dogs, 18 affected by KCS and 6 with unremarkable eyes were evaluated. Each dog underwent an ophthalmological examination including evaluation of the STT for each eye. A Rose Bengal-impregnated strip was moistened and applied to the eye. Staining was photographed and images were later evaluated by ten observers blinded to the KCS status of the eye. The percentage of the ocular surface retaining dye was scored as :0- no staining evident; 1 - $<20 \%$ of visible surface stained; $2-20-40 \%$; 340-60\%; 4- 60-80\%; 5- >80\%. The degree of staining was correlated with the STT value for each eye.

In the normal eyes, the mean STT was $18.2 \pm 2.9 \mathrm{~mm} / \mathrm{min}$ (range: $15-22 \mathrm{~mm} / \mathrm{min}$ ) and no eyes showed RBS retention. In the KCS group the mean STT result was $6.3 \pm 5.2$ (range 0-13 $\mathrm{mm} / \mathrm{min}$ ) and mean RBS score was $3.05 \pm 1.3$ (range 0.9-5). Mean responses between scorers varied up to $0.8 \pm 0.4$ points from the total mean, however correlation of responses for individual eyes was strong, with a minimum $R$ value of 0.70 and maximum of 0.93 . Further analysis showed a moderate negative correlation between RBS and STT (Pearson's Correlation Coefficient, R: -0.62, R2: 0.3924 , $\mathrm{P}=0.0001)$. The variation in results was broader at the lowest STT results, and further analysis separated eyes into those with STT values of 5 and above, and those below. For STT $<5 \mathrm{~mm} / \mathrm{min}, \mathrm{R}:-0.069$ (not significant at $\mathrm{P}<0.05$ ), whereas for those $5 \mathrm{~mm} / \mathrm{min}$ or above $\mathrm{R}$ : $-0.824, \mathrm{P}=0.00003$.

These results show that RBS correlates well with STT for dogs with eyes experiencing mild KCS but not for eyes with more severe KCS and STT $<5 \mathrm{~mm} / \mathrm{min}$. Further research is required to determine the value of RBS as a marker for canine ocular pathology.

\section{Variation in the treatment of canine acute glaucoma}

\section{Marnie Brennan', Martin Downes ${ }^{4}$, Gareth Jones $^{3}$, Rachel Dean ${ }^{1}$, Corinne Wigfall ${ }^{5}$}

1 Centre for Evidence-based Veterinary Medicine, University of Nottingham, Leicestershire, UK

Fremont Veterinary Clinic, Portland, Oregon, USA

The Park Veterinary Group, Leicestershire, UK

Griffith University, Nathan, Australia

Massey University, Palmerston North, New Zealand

Acute canine glaucoma is a highly morbid condition encountered by veterinarians yet there is little peer-reviewed research published comparing the most effective ways of treating important small animal ophthalmological conditions. Current treatment patterns of glaucoma are poorly documented. Establishing current practice would add to the existing knowledge base, but also identify areas of priority for research in veterinary ophthalmology. The aim of this study was to investigate the current treatment of acute glaucoma by first opinion veterinarians (GP) and those with a special interest in ophthalmology (SI) for both the purposes of comparison and benchmarking.

Cross sectional surveys using clinical vignettes were administered to GPs via a mailing list of the Centre for Evidence-based Veterinary Medicine (CEVM) and to SI attendees of the British Association of Veterinary Ophthalmologists (BrAVO) conference in 2011. A case of acute canine glaucoma was presented and respondents were asked for treatment recommendations using open-ended questions.

Of $267 \mathrm{GP}$ and $70 \mathrm{SI}$ respondents, the majority recommended topical carbonic anhydrase inhibitors (GP 56.9\%, SI $70 \%)$. The SI group were more likely to recommend a prostaglandin analogue (GP $27.7 \%$, SI $80.0 \%$ ), use of timolol either alone or in combination with a carbonic anhydrase inhibitor (GP 10.4\%, SI 20.0\%), and a topical steroid (GP $6.2 \%$, SI 20.0\%) than GPs. GPs recommended a miotic (GP $11.5 \%$, SI $0.0 \%)$ more frequently. A minority of both groups (15.4\%) suggested the use of systemic drugs to lower intraocular pressure (usually mannitol or oral carbonic anhydrase inhibitors). Analgesia (e.g. NSAIDs) was suggested by most respondents (GP 54.2\%, SI 54.3\%). 61.4\% of GPs indicated a desire to refer glaucoma to an ophthalmic specialist either acutely or for long term management. Median times to first recommended recheck for outpatient treatment were not significantly different for the two groups (GP 1.5 days; SI 2.0 days). A larger proportion of SI recommended hospitalization until normalization of intraocular pressure (GP 4.6\%, SI 35.7\%).

These results suggest moderate variation amongst practitioners in the treatment of acute canine glaucoma, especially with regard to the differences in the use of topical prostaglandins between GPs and SI. Differences may be the result of a lack of published information, clinical uncertainty, differences in case presentation or therapeutic costs/availability. These data contribute to the scarce knowledge base for the management for acute glaucoma. 